\title{
Synthesis of 2-Chloro-3-(2-naphthyloxy)quinoxaline
}

\section{Craig A. Obafemi* ${ }^{1}$, Wolfgang Pfleiderer ${ }^{2}$}

${ }^{1}$ Department of Chemistry, Obafemi Awolowo University, Ile-Ife, Nigeria

${ }^{2}$ Fachbereich Chemie, Konstanz University D-78457 Konstanz/ Germany

E-mail: adeyemi01@yahoo.com (craigoba@oauife.edu.ng)

*Author to whom correspondence should be addressed

Received: 7 February 2005 / Accepted: 2 February 2006 / Published: 1 December 2006

Keywords: 2,3-Dichoroquinoxaline, 2-naphthol.<smiles>Clc1nc2ccccc2nc1Cl</smiles><smiles>Oc1ccc2ccccc2c1</smiles>

2

1

\section{pyridine}

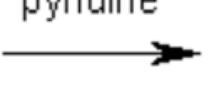

3

A mixture of 2,3-dichloroquinoxaline 1 [1] (1.0 g, $5 \mathrm{mmol})$ and 2-naphthol (0.72 g, $5 \mathrm{mmol})$ in pyridine $(10 \mathrm{~mL})$ was heated to $130^{\circ} \mathrm{C}$ for $3 \mathrm{~h}$, with magnetic stirring, to give a dark-brown reaction mixture. The reaction mixture was allowed to cool and then poured into cold water (50 $\mathrm{mL}$ ). The resulting solid was filtered, dried and purified by column chromatography on silica gel, eluting with petroleum ether (b.p. 100 ) / ethanol (100:1) to afford 2-chloro3-(2-naphthyloxy)quinoxaline 3 as colorless crystals (0.9 g, 58.0\%).

Melting point: $163-165^{\circ} \mathrm{C}$

${ }^{1}$ H-NMR (400 MHz, DMSO-d6, d (ppm): 8.10 - 7.90 (m, 5H, Ar-H), 7.76 - 7.67 (m, 3H, Ar-H), $7.62-7.54$ (m, 3H, Ar-H).

${ }^{13}$ C-NMR (100 MHz, DMSO-d6, d (ppm): 152.8, 150.1, 138.8, 138.6, 133.5, 131.0, 130.8, 129.6, 128.5, 127.7, 127.6, 127.4, 126.8, 126.6, 125.8, 121.5, 118.1.

MS (m/z, \%): $308\left([\mathrm{M}+2]^{+}\right.$31.8), $306\left(\mathrm{M}^{+}, 100\right), 278\left([\mathrm{M}-\mathrm{CO}]^{+}, 19.0\right), 271\left([\mathrm{M}-\mathrm{Cl}]^{+}, 23.6\right)$, $243\left([\mathrm{M}-\mathrm{CO}-\mathrm{Cl}]^{+}, 34.6\right), 163\left(\left[\mathrm{M}-\mathrm{C}_{10} \mathrm{H}_{7} \mathrm{O}\right]{ }^{+}\right.$, 8.6), 127 (17.3), 115 (50.3), 102 (45.6), 90 (12.0), 75 (22.2). 
We thank the Alexander von Humboldt Foundation for a post-doctoral fellowship (C.A.O.).

\section{Reference}

1. C.A. Obafemi and Wolfgang Pfleiderer, Helv. Chim. Acta 1994, 77, 1549 - 1556.

(C) 2006 MDPI. All rights reserved. 